\title{
Sialylation of Helicobacter bizzozeronii lipopolysaccharides modulates Toll-like receptor (TLR) 2 mediated response
}

\author{
Pradeep Kumar Kondadi, Joana Revez, Marja-Liisa Hänninen and Mirko Rossi*
}

\begin{abstract}
Sialic acid in lipopolysaccharides (LPS) of mucosal pathogens is known to be an important virulence factor. Few strains of Helicobacter pylori express sialyl-Lewis-X and we have reported that human and canine Helicobacter bizzozeronii strains express sialyl-lactoseamine in their LPS. However, the role of sialyation of Helicobacter LPS in the interaction with the host cells is still unknown. In this study H. bizzozeronii LPS is shown to activate the TLR2 in a dose and strain dependent manner in the in vitro HEK-293 cells model expressing TLR2, but not the cells expressing TLR4. These results indicate that TLR2 is the specific receptor for $H$. bizzozzeronii LPS, as previously described for $H$. pylori. To further explore the role of sialylation of $H$. bizzozeronii LPS on TLR2 response, $H$. bizzozeronii $\Delta$ hbs 2 mutant strains deficient in sialyltransferase activity were constructed by homologous recombination. LPS from $\mathrm{H}$. bizzozeronii $\Delta$ hbs2 strains enhanced the NF-kB induction via TLR2 compared to the respective wild types, leading to the conclusion that the sialylation of H. bizzozeronii LPS in wild-type strains may modulate host immune response.
\end{abstract}

\section{Introduction}

In both humans and several animal species helicobacters are recognised as an etiologic agent of chronic gastritis, gastric ulcers and, in some cases, even gastric adenocarcinoma and MALT lymphoma [1,2]. Helicobacter spp. are highly diverse, appear to have a host species-specific tropism, the transmission occurs mainly vertically and the colonisation generally persists throughout the lifetime of their hosts $[1,2]$. Several species have also developed the ability to jump between different hosts [1,3]. Among them, the gastric Helicobacter spp., belonging to the group of $H$. heilmannii sensu lato (including $H$. bizzozeronii, $H$. felis, $H$. suis and $H$. heilmannii sensu stricto), have zoonotic potential and have been detected in $0.6-2 \%$ of human gastritis $[1,3]$.

After penetration of the mucous layer, gastric epithelial cells provide the first point of contact between helicobacters and their hosts [4]. By recognizing diverse microbial products, Toll-like receptors (TLRs) expressed on the surface of gastric epithelial cells play a key role in mediating cell-signalling which results in the induction

\footnotetext{
* Correspondence: mirko.rossi@helsinki.fi

Department of Food Hygiene and Environmental Health, Faculty of Veterinary Medicine, University of Helsinki, P.O. Box 66, Agnes Sjöbergin katu 2, Fl-00014 Helsinki, Finland
}

of host defence stimulation [5]. Although all described TLRs have the ability to activate the key transcription factors NF- $\mathrm{kB}$ and AP-1, there are differences in the ultimate gene-expression profile that results from the activation of a specific TLR [6]. For example, it has been described that TLR2 and TLR4 differentially activate human dendritic cells (DC) resulting in differences in cytokine and chemokine gene transcription, suggesting that TLR2 and TLR4 signalling is not equivalent [7]. TLR4 specifically recognizes Lipopolysaccharide (LPS) from Gram negative bacteria [8]. TLR2 has a broader spectrum of ligands including also forms of LPS that are structurally different from those recognized by TLR4 [6,8]. In particular, it has been described that $H$. pylori LPS specifically stimulate TLR2 and act as antagonist of TLR4 [6,9]. Very little is known about the ability of non-H. pylori Helicobacter species LPS in modulating host-response, despite the fact they express different LPS structures $[10,11]$. Actually, the human pathogen canine-adapted H. bizzozeronii expresses phase variable sialyl-lactoseamine [11]: a feature not detected in $H$. pylori which rarely express sialyl-Lewis antigens on their LPS. Therefore, the aim of this paper was to elucidate the interaction between $H$. bizzozeronii LPS and human TLR as first stage in host-bacterial 
recognition, focusing in particular on understanding the role of sialylated LPS in this interaction.

\section{Material and methods Cell lines}

HEK-293 cells from InvivoGen (Toulouse, France) stably transfected with human TLR-2 (HEK-Blue ${ }^{\text {Tw- }}$ hTLR2), and TLR4 (HEK-Blue $\left.{ }^{\mathrm{Tm}}-\mathrm{hTLR} 4\right)$ and HEK-Blue ${ }^{\mathrm{Tm}}$ Null1 cells non-expressing either TLR2 or TLR4 were grown and maintained according to the manufacturer's guidelines. Briefly, HEK-Blue ${ }^{\mathrm{Tw}}$ - cell lines were cultured in DMEM (Life Technology, Carlsbad, CA, USA) containing 10\% Fetal Bovine Serum (FBS) supplemented with 50 U.mL ${ }^{-1}$ of penicillin and $50 \mu \mathrm{g} \cdot \mathrm{mL}^{-1}$ of streptomycin (Life Technology), $100 \mu \mathrm{g} \cdot \mathrm{mL}^{-1}$ of normocin (InvivoGen), and $1 \mathrm{X}$ HEK-Blue $^{\text {th }}$ selection (InvivoGen; selection for HEKBlue $^{\text {tm }}$-hTLR2 and HEK-Blue ${ }^{\text {tm }}$-hTLR4) or $100 \mu \mathrm{g} \cdot \mathrm{mL}^{-1}$ zeocin (InvivoGen; selection for HEK-Blue ${ }^{\text {tix }}$ Null1).

\section{Bacterial strains and growth conditions}

Bacterial strains used in this study are listed in Table 1 . Helicobacter strains were routinely grown on HP medium (LabM Limited, Lancashire, UK) containing 5\% bovine blood and Skirrow selective supplement (Oxoid, Ltd., Cambridge, UK) at $37{ }^{\circ} \mathrm{C}$ in an incubator with microaerobic atmosphere (Thermo Forma, Series II Water Jacketed Incubator; Thermo Fisher Scientific, Waltham, MA, USA). For LPS extraction Helicobacter strains were cultivated in Brain Heart Infusion (BHI, BD, Becton, Dickinson and Co., NJ, USA) containing $10 \%$ of FBS, Skirrow selective supplement (Oxoid) and Vitox supplement (Oxoid) at $37{ }^{\circ} \mathrm{C}$ in a jar with microaerobic atmosphere. Campylobacter jejuni was

Table 1 Bacterial strains used in this study (h: human isolates; c: canine isolates).

\begin{tabular}{|c|c|c|}
\hline Bacterial species & Strain & Reference or source \\
\hline \multirow[t]{13}{*}{ Helicobacter bizzozeronii } & CIII-1 ${ }^{\text {GEN }}$ (h) & {$[12,13]$} \\
\hline & R53 (h) & {$[14]$} \\
\hline & Storkis CCUG $35545^{\top}$ (c) & {$[15]$} \\
\hline & 14 CCUG 35546 (c) & {$[15]$} \\
\hline & $12 \mathrm{a}(\mathrm{c})$ & {$[15]$} \\
\hline & $10 F(c)$ & {$[15]$} \\
\hline & Yrjälä (c) & {$[15]$} \\
\hline & Emo (c) & {$[15]$} \\
\hline & Heydar (c) & {$[15]$} \\
\hline & Heydar $\Delta$ hsb2-M2 & this study \\
\hline & Heydar $\Delta$ hsb2-M4 & this study \\
\hline & $\mathrm{Clll}-1^{\mathrm{GEN}} \Delta$ hsb2-M9 & this study \\
\hline & $\mathrm{ClIII-1}^{\mathrm{GEN}} \Delta$ hsb2-M13 & this study \\
\hline Helicobacter pylori & 26695 & [16] \\
\hline Campylobacter jejuni & $81-176$ & {$[17]$} \\
\hline
\end{tabular}

cultivated in Nutrient Agar (Oxoid) supplemented with 5\% of bovine blood.

\section{H. bizzozeronii a2,3-sialyltransferase gene (hbs2) mutant strains}

In $H$. bizzozeronii strains CIII-1 (human isolate) and Heydar (canine isolate), chromosomal inactivation of $\alpha 2,3$-sialyltransferase gene ( $h b s 2$ ) was performed by allelic exchange using the chloramphenicol resistance gene (cat), as previously described [18]. The cat gene was introduced in the same direction as the target gene using $X b a \mathrm{I}$ and $K p n I$ restriction sites. The resultant plasmid, pMRS3, was constructed and amplified in E. coli TOPO10 and used as a suicide plasmid in $H$. bizzozeronii. Mutants were obtained by electroporation as described for $H$. felis [19]. After electroporation, the bacteria were left to recover on $\mathrm{HP}$ agar plates for $48 \mathrm{~h}$ under microaerobic conditions as described above. The mutant strains were selected on HP agar plates supplemented with $10 \mathrm{mg} \cdot \mathrm{mL}^{-1}$ of chloramphenicol (Sigma-Aldrich, St. Louis, MI, USA). The plates were incubated for up to 10 days, and the site of recombination was verified by PCR and sequencing.

\section{LPS extraction and LPS SDS-PAGE profile}

LPS was extracted from biomass obtained after $48 \mathrm{~h}$ of incubation in BHI broth. Crude LPS was extracted by using the hot phenol-water method, and subsequent purification by enzymatic treatments (RNase A, DNase II and proteinase $\mathrm{K}$ ) as described previously [6]. LPS were treated with Lysozyme to remove traces of peptidoglycan contamination [6]. After the enzymatic treatments, the LPS was precipitated at $-20{ }^{\circ} \mathrm{C}$ overnight in 10 volumes of pure ethanol in presence of $0.03 \mathrm{M}$ of sodium acetate and re-suspended in water and the concentration was then determined by purpald assay [20]. The LPS obtained was essentially free of proteins and nucleic acids, and it had an electrophoretic profile similar to that previously reported for the low-molecular-mass $H$. bizzozeronii LPS [11]. LPS was treated overnight with $6.7 \mathrm{U} \cdot \mathrm{mL}^{-1}$ of neuraminidase from Clostridium perfringens (SigmaAldrich) at pH 6. LPS neuraminidase treated and untreated samples were loaded on 15\% TRIS-Glycine SDS-PAGE (Biorad, Hercules, CA, US), run for $2 \mathrm{~h}$ and $50 \mathrm{~min}$ at constant $20 \mathrm{~mA}$ and then silver stained as previous described [11].

\section{NF-KB stimulation using HEK-Blue ${ }^{\mathrm{TM}}$ system and IL-8 determination}

HEK-Blue ${ }^{\mathrm{TM}}$ cell lines are engineered HEK293 cells stably transfected with a vector expressing secreted embryonic alkaline phosphatase reporter (SEAP) gene under the control of an inducible NF-kB promoter. Therefore, stimulation of TLRs will result in an amount of extracellular SEAP in the supernatant that is proportional to the 
level of NF-kB induction. For the determination of NF$\mathrm{\kappa B}$ stimulation, 96-well plates were seeded with $5.0 \times 10^{4}$ cells/well of HEK-Blue ${ }^{\text {Tw }}$-hTLR2 or HEK-Blue ${ }^{\text {Tu }}$ Null1, or $2.5 \times 10^{4}$ cells/well of HEK-Blue ${ }^{\mathrm{tm}}$-hTLR4. After overnight incubation, cells were treated for $24 \mathrm{~h}$ with different concentrations of LPS extracted from $H$. bizzozzeronii strains. SEAP was measured at $\mathrm{OD}_{620}$ after $1 \mathrm{~h}$ of incubation at $37^{\circ} \mathrm{C}$, by the addition of $180 \mu \mathrm{L}$ of QUANTIBlue $^{\text {tix }}$ (InvivoGen) to $20 \mu \mathrm{L}$ of the HEK-Blue ${ }^{\text {tm }}$ cells supernatants [21]. For some experiments LPS was pretreated with $40 \mu \mathrm{g} \cdot \mathrm{ml}^{-1}$ polymyxin $\mathrm{B}$ sulphate (PB; Sigma-Aldrich) at $37{ }^{\circ} \mathrm{C}$ for $45 \mathrm{~min}$. As positive control for HEK-Blue $\mathrm{e}^{\mathrm{Tw}}$-hTLR2, HEK-Blue ${ }^{\mathrm{Tw}}-$ hTLR4 and HEK-Blue $\mathrm{e}^{\mathrm{Tw}}$ Null1, $100 \mathrm{ng} \cdot \mathrm{mL}^{-1}$ of Pam2CSK4 (Invivogen), $5 \mathrm{ng} \cdot \mathrm{mL}^{-1}$ of E. coli LPS (Sigma-Aldrich) and $50 \mathrm{ng} / \mathrm{mL}$ of Polyinosinic-polycytidylic acid (Invivogen) were used, respectively. After $24 \mathrm{~h}$ incubation, induced HEK-Blue ${ }^{\mathrm{rm}}-$ hTLR2 supernatant was collected and IL-8 expression was determined by ELISA according to the manufacturer's guidelines (DuoSet ELISA development system: $R \& D$ systems). All experiments were done in triplicate.

\section{Statistical analysis}

Statistical analysis was performed using GraphPad Prism version 6 for Windows, (San Diego California USA). For groups comparison one-way ANOVA followed by Bonferroni post-test with a cut-off of 0.05 was selected, while for pairwise comparison unpaired two-tailed $t$-test was carried out. For trend analysis one-way ANOVA was followed by the test for linear trend as implemented in the software. Error bars in the graphs in all figures were calculated as Standard Error of the Mean (SEM).

\section{Results}

To identify the TLR responsible for the recognition of $H$. bizzozzeronii LPS, HEK-293 cells stably transfected with human TLR-2 (HEK-Blue ${ }^{\text {tm }}$-hTLR2), and TLR4 (HEKBlue $^{\mathrm{m}}$-hTLR4) and HEK-Blue ${ }^{\mathrm{Tm}}$ Null1 cells non-expressing either TLR2 or TLR4 were stimulated with $25 \mu \mathrm{g} \cdot \mathrm{mL}^{-1}$ of LPS extracted from several human and canine H. bizzozzeronii strains. $H$. bizzozzeronii LPS showed to activate only the HEK-Blue ${ }^{\mathrm{m}}$-hTLR2 in a strain dependent manner (Figure 1). The NF- $\mathrm{kB}$ induction in HEK-Blue ${ }^{\mathrm{Tm}}-\mathrm{hTLR}$ 4 or HEK-Blue $^{\text {tw }}$ Null1 cells treated with $H$. bizzozeronii LPS was not different from untreated cells $\left(\mathrm{OD}_{620}, 0.108 \pm\right.$ 0.03). For further analysis, two $H$. bizzozeronii strains inducing high $\left(\mathrm{CIII}-1^{\mathrm{GEN}}\right)$ and low (Heydar) NF- $\mathrm{kB}$ were selected. NF- $\mathrm{\kappa B}$ induction in HEK-Blue ${ }^{\mathrm{Ts}}$-hTLR2 cells in response to $H$. bizzozeronii $\mathrm{CIII-1}{ }^{\mathrm{GEN}}$ and Heydar LPS was found to be dose dependent (one-way ANOVA, test for linear trend, $p<0.05$; Figure $2 \mathrm{~A}$ ). To demonstrate that the observed TLR2-mediated effects were LPS specific, LPS was pre-incubated with the antibiotic polymyxin B sulphate $(\mathrm{PB})$, an inhibitor of the activating properties of
LPS [6]. Therefore, $12.5 \mu \mathrm{g} \cdot \mathrm{mL}^{-1}$ of LPS from H. bizzozzeronii CIII- $1^{\mathrm{GEN}}$ and Heydar were pre-treated with $40 \mu \mathrm{g} \cdot \mathrm{mL}^{-1}$ of $\mathrm{PB}$ at $37^{\circ} \mathrm{C}$ for $45 \mathrm{~min}$ before infecting the HEK-Blue ${ }^{\text {tw }}$-hTLR2. A significant difference in the NF- $\mathrm{KB}$-inducing ability of LPS before and after treatment was observed for both stains (unpaired two-tailed $t$-test $p<0.05$; Figure $2 \mathrm{~B}$ ), indicating that TLR2 activation was mediated by $H$. bizzozzeronii LPS. PB was able to inhibit the TLR2 activation only partially. This is a consequence of low extent of phosphorylation in the lipid A of Helicobacter spp. LPS which is required for binding $\mathrm{PB}$, as previously described [6]. In order to study the effect of sialylation of $H$. bizzozeronii LPS on TLR2 response, $H$. bizzozeronii $\Delta h b s 2$ mutant strains deficient in sialyltransferase activity were created. The LPS profile of CIII- ${ }^{\text {GEN }}$ wild type and Heydar wild type showed low-molecular-weight LPS with a clear switch of the band after neuraminidase treatment but no switch was observed in the CIII- ${ }^{\text {GEN }} \Delta h b s 2$ and Heydar $\Delta h b s 2$ mutants as we expected (see Additional file 1). HEK-Blue ${ }^{\text {tw }}$-hTLR2 were incubated for $24 \mathrm{~h}$ with different concentrations of LPS isolated from CIII- ${ }^{\text {GEN }}$ wild type, Heydar wild type and corresponding $\Delta h b s 2 \mathrm{mu}-$ tant strains (Figure 3). H. bizzozeronii CIII-1 ${ }^{\text {GEN }} \Delta$ hbs2M9 and Heydar $\Delta h b s 2-M 4$ mutant strains enhanced significantly the induction of NF-kB compared to wild type strains (unpaired two-tailed $t$-test $p<0.05$; Figure 3A). To further explore IL-8-inducing activity of $H$. bizzozeronii LPS, supernatants of HEK-Blue ${ }^{\text {max }}$-hTLR2 were collected after $24 \mathrm{~h}$ of incubation with different concentrations of LPS isolated from CIII- ${ }^{\text {GEN }}$ wild type, Heydar wild type and corresponding $\Delta h b s 2$ mutant strains and IL-8 expression was determined by DuoSet ELISA Kit. The disruption of $h b s 2$ in H. bizzozeronii significantly enhanced the expression of IL-8 HEK-Blue ${ }^{\text {tm }}$ hTLR2 cells (unpaired two-tailed $t$-test $p<0.05$; Figure $3 \mathrm{~B}$ ). To verify if that increase of IL-8 expression by HEK-Blue ${ }^{\text {tix }}$-hTLR2 was a result of the mutation of $h b s 2$ and not due to a polar effect, and in the absence of a protocol for the complementation, two independent isogenic mutants were selected one from each $\mathrm{H}$. bizzozeronii strains: CIII- ${ }^{\mathrm{GEN}} \Delta h b s 2-\mathrm{M} 13$ and Heydar $\Delta h b s 2-M 2$. After inoculating HEK-Blue ${ }^{\text {twx }}$-hTLR2 cells with $12.5 \mu \mathrm{g} \cdot \mathrm{mL}^{-1}$ of LPS we observed that both isogenic $H$. bizzozeronii CIII-1 ${ }^{\text {GEN }} \Delta h b s 2-\mathrm{M} 13$ and Heydar $\Delta h b s 2-\mathrm{M} 2$ mutants enhanced the expression of IL- 8 by 2.4 and 3.0 fold, compared to the respective wild type strain. Although we observed a lower increase of IL-8 expression compared to $H$. bizzozeronii CIII- ${ }^{\text {GEN }}$ $\Delta h b s 2-\mathrm{M} 9$ (5.4 fold) and Heydar $\Delta h b s 2-\mathrm{M} 4$ (4.8 fold) mutants, these results confirmed the role of core or Ochain composition and particularly the presence of sialic acid in the modulation of the host response through TLR2 activation. 


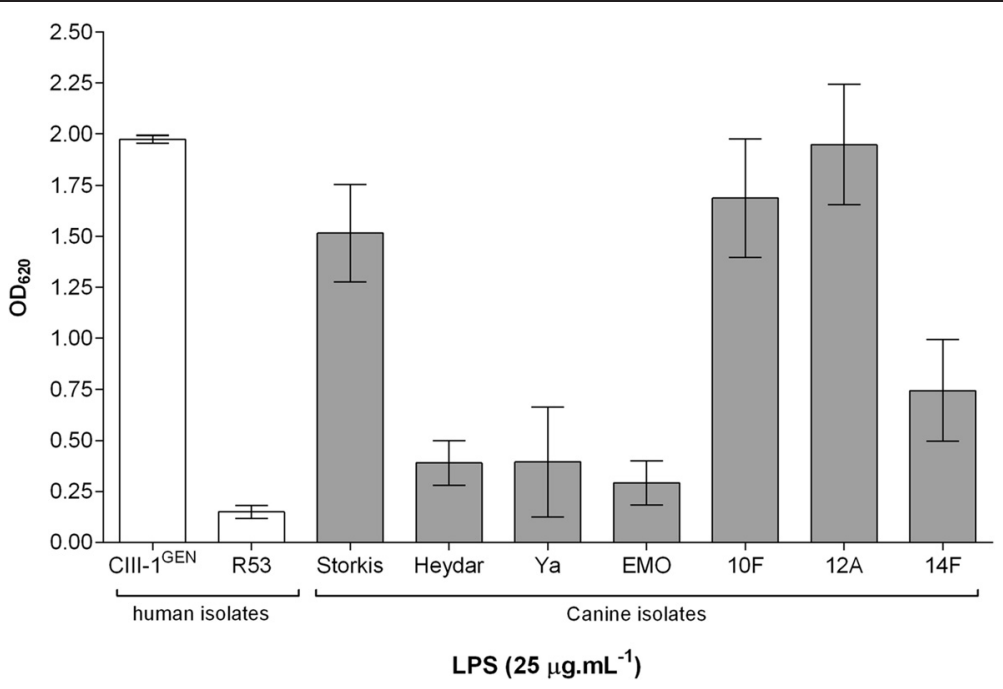

Figure 1 Differential human TLR2 activation by $\boldsymbol{H}$. bizzozeronii LPS. HEK-Blue ${ }^{\mathrm{TM}}-\mathrm{hTLR} 2$ cells were incubated for $24 \mathrm{~h}$ with $\mathrm{LPS}\left(25 \mu \mathrm{g} \cdot \mathrm{mL}{ }^{-1}\right.$ ) extracted from different human (white bars) and canine (grey bars) strains, and NF-kB-induced SEAP activity was assessed using QUANTI-Blue ${ }^{\mathrm{TM}}$ and by reading the $\mathrm{OD}$ at $620 \mathrm{~nm}$. The values were normalized by subtracting the average $\mathrm{OD}_{620}$ values of the untreated cells. The OD 620 value of the positive control Pam2CSK4 was $2.25 \pm 0.12$. Error bars show \pm SEM.

\section{Discussion}

There have been conflicting findings in the literature concerning the TLR responsible for the recognition of Helicobacter LPS, as some authors have suggested that Helicobacter LPS stimulate TLR4, whereas others proposed a role for TLR2 [6,22]. Although we cannot completely exclude possible contaminations, the absence of NF- $\mathrm{kB}$ induction in either HEK-Blue ${ }^{\mathrm{Tw}}-\mathrm{hTLR} 4$ or HEKBlue $^{\mathrm{Tx}}$ Null1 cells, the suppression effect of PB on the TLR2 activation and the dose-depend response of HEKBlue $^{\mathrm{mx}}$-hTLR2 suggest that $H$. bizzozeronii LPS function as a classic TLR2 ligand, as described for H. pylori [6] and for $H$. felis [22]. We observed a degree of variation in the TLR2 mediated NF-kB -inducing ability of LPS prepared from different strains of $H$. bizzozeronii. As previously described, these differences could be the consequence of variations in the degree of acylation and/or phosphorylation of the LPS from different isolates [6]. However, MALDI-TOF analysis indicated that Lipid A structures from both the selected $H$. bizzozeronii strains CIII- $1^{\text {GEN }}$ (inducing high concentration of NF- $\mathrm{kB}$ ) and Heydar (inducing low concentration of NF- $\mathrm{kB}$ ) were
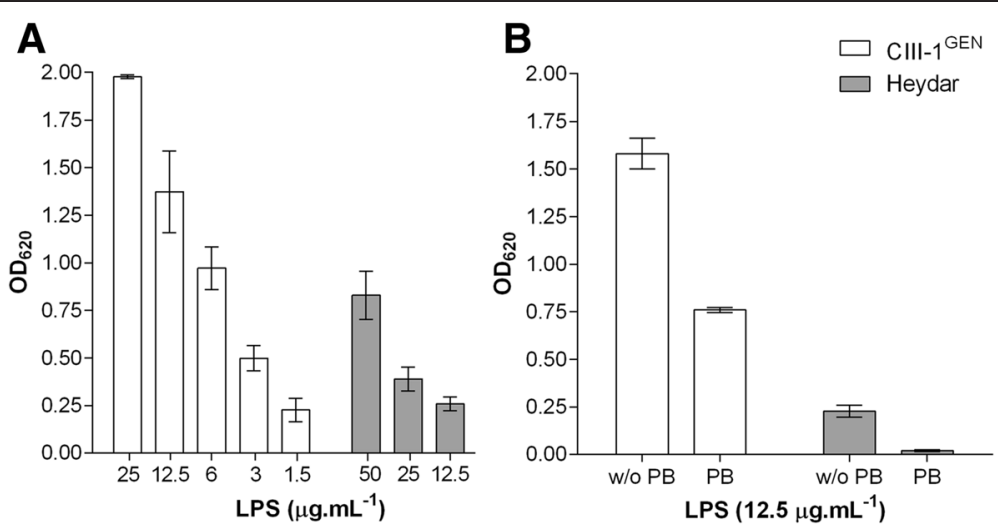

Figure 2 Human TLR2 response is mediated by H. bizzozzeronii LPS. (A) H. bizzozeronii LPS activates human TLR2 in dose dependent manner. HEK-Blue ${ }^{\mathrm{TM}}$-hTLR2 cells were incubated for $24 \mathrm{~h}$ with different concentrations of LPS from human isolate CIII-1 ${ }^{\mathrm{GEN}}$ (white bars) and canine isolate Heydar (grey bars) H. bizzozzeronii strains. HEK-Blue ${ }^{T M}-h T L R 2$ response decrease significantly (test for linear trend; $p<0.05$ ). Error bars show \pm SEM. (B) Inhibitory effect of Polymyxin B in TLR2 activation by H. bizzozzeronii LPS. LPS $\left(12.5 \mu \mathrm{g} \cdot \mathrm{mL}^{-1}\right)$ extracted from the human strain CIII- 1 GEN (white bars) and the canine Heydar (grey bars) H. bizzozzeronii strains, were pre-incubated with PB $\left(40 \mu \mathrm{g} \cdot \mathrm{mL}^{-1}\right)$ for $45 \mathrm{~min}$ at $37^{\circ} \mathrm{C}$. HEK-Blue ${ }^{\mathrm{TM}}$-hTLR2 cells were incubated with LPS (with and without PB treatment) for $24 \mathrm{~h}$. HEK-Blue ${ }^{\mathrm{TM}}$-hTLR2 response was inhibited in both strains after treatment with PB and the response was significantly different $(p<0.05)$ in both the strains. The values were normalized by subtracting the average $\mathrm{OD}_{620}$ values of the untreated cells. The $\mathrm{OD}_{620}$ value of the positive control Pam2CSK4 was $2.25 \pm 0.12$. Error bars show \pm SEM. 


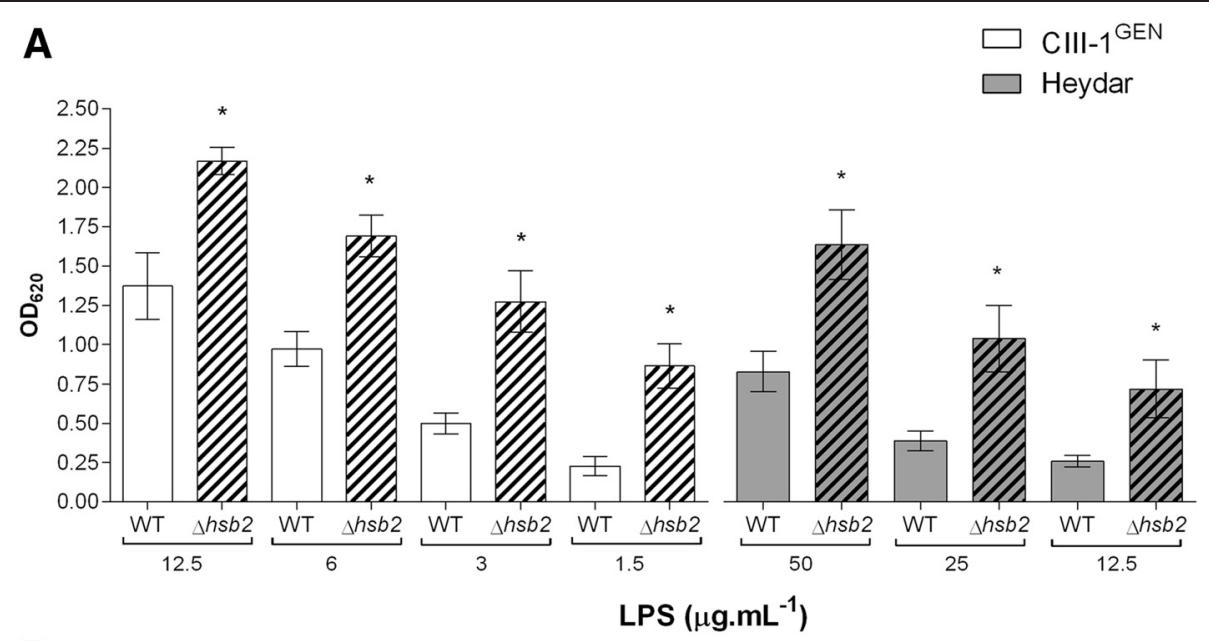

B

\section{LPS $\left(\mu \mathrm{g} \cdot \mathrm{mL}^{-1}\right)$}

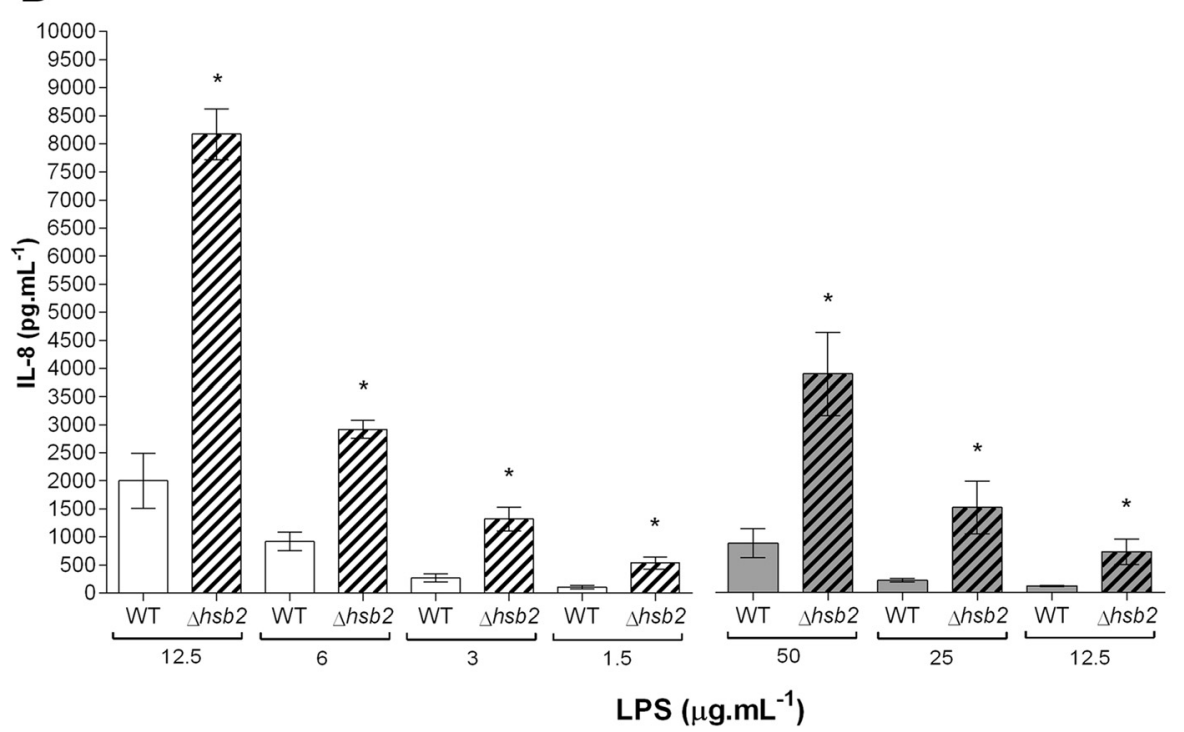

Figure 3 Desialylated LPS of $\boldsymbol{H}$. bizzozeronii increases human TLR2 response. (A) NF-KB stimulation and (B) IL-8 expression by HEK-Blue ${ }^{\text {TM }}$ hTLR2 cells were determined after $24 \mathrm{~h}$ of incubation with different concentrations of LPS isolated from CIII-1 ${ }^{\mathrm{GEN}}$ wild type (white bars), Heydar wild type (grey bars) and respective $\Delta$ hbs2 mutants (striped bars). The TLR2 response between wild type and mutants was significantly different $\left({ }^{*} p<0.05\right)$, respectively. Error bars show \pm SEM.

identical, resembling that of H. pylori [23] in which 3hydroxyoctadecanoic acid, n-octadecanoic acid, and 3hydroxyhexadecanoic acid are substituting at $\mathrm{O}-$ and $\mathrm{N}$ positions (see Additional file 2). In addition, phosphoethanolamine was shown to be present in the Lipid A of both $H$. bizzozzeronii strains and no extra phosphates were detected (data not shown). Therefore, in agreement with previous studies $[6,22]$, we hypothesized that also differences in the core and O-chains of the LPS from individual isolates could modulate the IL-8-inducing activity of the lipid A component [22]. In fact, although both $H$. bizzozeronii CIII- ${ }^{\text {GEN }}$ and Heydar strains have a single copy of $\alpha 2,3$-sialyltransferase gene ( $h b s 2)$ and express sialic acid on their LPS, they shown different reactivity with cholera toxin, indicating possible different LPS structures [11]. Studies on C. jejuni have shown that sialylation of lipooligosaccharides (LOS) modulates the dendritic cells (DC) response via TLR4 [24]. In particular, the presence of sialic acid on the $C$. jejuni LOS induced a stronger DC activation and subsequent $\mathrm{B}$ cell proliferation than did desialylated LOS by increasing TLR4-mediated signalling [24]. These data together with the results from another study [25] indicate that not only the lipid A structure but also the C. jejuni LOS carbohydrate moiety modulates TLR4-mediated host-response. Similarly, in the present study we showed that TLR2-mediated NF- $\mathrm{kB}$ induction and resulting IL-8 expression in HEK293 was modulated by the H. bizzozeronii LPS carbohydrate moiety and in particular by the presence of sialic acid. However, differently to what is 
observed in TLR4, sialylation seems to inhibit TLR2mediated induction of NF-kB.

It has been suggested that TLR2 on DCs plays an important role in immune tolerance [26]. However, it was also showed that TLR2 on epithelial cells activates inflammatory mediators [6]. Thus, activation of TLR2, on the one hand, increases the immune tolerance favouring persistence of the bacterium in the stomach and, on the other hand, increases epithelial inflammatory responses resulting potentially in more severe gastritis [26]. However, Sun et al. observed enhanced gastric immunopathology in $\mathrm{H}$. pylori-infected TLR2-KO mice, indicating that the impact of total TLR2 deficiency is greater on immune cells than on epithelial cells [26]. Therefore, it is tempting to speculate that the sialylation of $H$. bizzozeronii LPS may increase inflammatory responses by depressing the TLR2 response. However, further in vivo studies are needed for elucidating the proinflammatory effect of Helicobacter sialyl-LPS.

In conclusion, our study showed that the sialylation of $H$. bizzozeronii LPS in wild-type strains may modulate host immune response. Since we observed that the expression of sialylated LPS by $H$. bizzozeronii undergoes phase and phenotypic variation [11], changes in the stomach microenvironments due to diet, host-jump, antimicrobial treatment [12], may select subpopulations of $H$. bizzozeronii expressing or not sialylated LPS. These events could lead to an imbalance in the relationship between $H$. bizzozeronii and his host, underpinning the development of gastritis in both dogs and humans.

\section{Additional files}

\section{Additional file 1: LPS profile of wild type and mutant $H$.}

bizzozeronii CIII-1 ${ }^{\text {GEN }}$ and Heydar strains. LPS profile in 15\% TRIS-Glycine

SDS-PAGE gel. H. bizzozzeronii strains CIII-1 ${ }^{\mathrm{GEN}}$ wild type and Heydar wild type showed low-molecular-weight LPS with a clear switch of the band after neuraminidase treatment $\left(^{*}\right)$ but no switch was observed in the relative $\Delta h$ bs2 isogenic mutants.

Additional file 2: MALDI-TOF spectra of $H$. bizzozeronii CIII- ${ }^{\text {GEN }}$ wild type and Heydar wild type LPS. (A) H. bizzozeronii Clll-1 ${ }^{\mathrm{GEN}}$. (B) H. bizzozeronii Heydar.

\section{Abbreviations}

TLR: Tool-like Receptor; LPS: Lipopolysaccharide; PB: polymyxin-B.

\section{Competing interests}

The authors declare that they have no competing interests.

\section{Authors' contributions}

PKK carried out the experiments; JR participated in performing and coordinating the study, and draft the manuscript; MLJ helped to draft the manuscript; MR conceived of the study, and participated in its design and coordination, constructed the $\mathrm{H}$. bizzozeronii mutants and draft the manuscript. All authors read and approved the final manuscript.

\section{Acknowledgements}

This work was supported by the Academy of Finland (FCoE MiFoSa, no. 118602 and 141140). MR. was supported by an Academy of Finland
Postdoctoral Fellowship (no. 132940). We are thankful to Dr Taija Pietilä and Prof. Airi Palva (University of Helsinki) for their technical support regarding HEK cells lines. We are also thankful to Prof. Elke Schweda (Linköping Univerisity) and Dr Li Jianjun (NRC, Canada) for performing the MALDI-TOF analyses on Helicobacter LPS.

Received: 7 May 2014 Accepted: 10 December 2014

Published online: 21 January 2015

\section{References}

1. Haesebrouck F, Pasmans F, Flahou B, Chiers K, Baele M, Meyns T, Decostere A, Ducatelle R: Gastric helicobacters in domestic animals and nonhuman primates and their significance for human health. Clin Microbiol Rev 2009, 22:202-223.

2. Atherton JC, Blaser MJ: Coadaptation of Helicobacter pylori and humans: ancient history, modern implications. J Clin Invest 2009, 119:2475-2487.

3. Schott T, Kondadi PK, Hanninen ML, Rossi M: Comparative Genomics of Helicobacter pylori and the human-derived Helicobacter bizzozeronii CIII-1 strain reveal the molecular basis of the zoonotic nature of non-pylori gastric Helicobacter infections in humans. BMC Genomics 2011, 12:534.

4. Uno K, Kato K, Atsumi T, Suzuki T, Yoshitake J, Morita H, Ohara S, Kotake Y, Shimosegawa T, Yoshimura T: Toll-like receptor (TLR) 2 induced through TLR4 signaling initiated by Helicobacter pylori cooperatively amplifies iNOS induction in gastric epithelial cells. Am J Physiol Gastrointest Liver Physiol 2007, 293:G1004-G1012.

5. Akira S, Takeda K: Toll-like receptor signalling. Nat Rev Immunol 2004, 4:499-511

6. Smith SM, Moran AP, Duggan SP, Ahmed SE, Mohamed AS, Windle H O'Neill LA, Kelleher DP: Tribbles 3: a novel regulator of TLR2-mediated signaling in response to Helicobacter pylori lipopolysaccharide. J Immunol 2011, 186:2462-2471.

7. Re F, Strominger JL: Toll-like receptor 2 (TLR2) and TLR4 differentially activate human dendritic cells. J Biol Chem 2001, 276:37692-37699.

8. Takeda K, Akira S: Toll-like receptors in innate immunity. Int Immunol 2005, 17:1-14

9. Lepper PM, Triantafilou M, Schumann C, Schneider EM, Triantafilou K: Lipopolysaccharides from Helicobacter pylori can act as antagonists for Toll-like receptor 4. Cell Microbiol 2005, 7:519-528.

10. Hynes SO, Ferris JA, Szponar B, Wadstrom T, Fox JG, O'Rourke J, Larsson L, Yaquian E, Ljungh A, Clyne M, Andersen LP, Moran AP: Comparative chemical and biological characterization of the lipopolysaccharides of gastric and enterohepatic helicobacters. Helicobacter 2004, 9:313-323.

11. Kondadi PK, Rossi M, Twelkmeyer B, Schur MJ, Li J, Schott T, Paulin L, Auvinen P, Hanninen ML, Schweda EK, Wakarchuk W: Identification and characterization of a lipopolysaccharide alpha,2,3-sialyltransferase from the human pathogen Helicobacter bizzozeronii. J Bacteriol 2012, 194:2540-2550.

12. Schott T, Kondadi PK, Hanninen ML, Rossi M: Microevolution of a zoonotic Helicobacter population colonizing the stomach of a human host before and after failed treatment. Genome Biol Evol 2012, 4:1310-1315.

13. Kivisto R, Linros J, Rossi M, Rautelin H, Hanninen ML: Characterization of multiple Helicobacter bizzozeronii isolates from a Finnish patient with severe dyspeptic symptoms and chronic active gastritis. Helicobacter 2010, 15:58-66.

14. Jalava K, On SL, Harrington CS, Andersen LP, Hanninen ML, Vandamme P: A cultured strain of "Helicobacter heilmannii," a human gastric pathogen, identified as H. bizzozeronii: evidence for zoonotic potential of Helicobacter. Emerg Infect Dis 2001, 7:1036-1038.

15. Jalava K, On SL, Vandamme PA, Happonen I, Sukura A, Hanninen ML: Isolation and identification of Helicobacter spp. from canine and feline gastric mucosa. Appl Environ Microbiol 1998, 64:3998-4006.

16. Tomb JF, White O, Kerlavage AR, Clayton RA, Sutton GG, Fleischmann RD, Ketchum KA, Klenk HP, Gill S, Dougherty BA, Nelson K, Quackenbush J, Zhou L, Kirkness EF, Peterson S, Loftus B, Richardson D, Dodson R, Khalak HG, Glodek A, McKenney K, Fitzegerald LM, Lee N, Adams MD, Hickey EK, Berg DE, Gocayne JD, Utterback TR, Peterson JD, Kelley JM, et al: The complete genome sequence of the gastric pathogen Helicobacter pylori. Nature 1997, 388:539-547.

17. Black RE, Levine MM, Clements ML, Hughes TP, Blaser MJ: Experimental Campylobacter jejuni infection in humans. J Infect Dis 1988, 157:472-479. 
18. Kondadi PK, Pacini C, Revez J, Hanninen ML, Rossi M: Contingency nature of Helicobacter bizzozeronii oxygen-insensitive $\mathrm{NAD}(\mathrm{P}) \mathrm{H}$-nitroreductase (HBZC1_00960) and its role in metronidazole resistance. Vet Res 2013, 44:56.

19. Josenhans C, Ferrero RL, Labigne A, Suerbaum S: Cloning and allelic exchange mutagenesis of two flagellin genes of Helicobacter felis. Mol Microbiol 1999; 33:350-362.

20. Lee $\mathrm{CH}$, Tsai CM: Quantification of bacterial lipopolysaccharides by the purpald assay: measuring formaldehyde generated from 2-keto-3deoxyoctonate and heptose at the inner core by periodate oxidation. Anal Biochem 1999, 267:161-168.

21. Douillard FP, Ribbera A, Jarvinen HM, Kant R, Pietila TE, Randazzo C, Paulin L, Laine PK, Caggia C, von Ossowski I, Reunanen J, Satokari R, Salminen S, Palva A, de Vos WM: Comparative genomic and functional analysis of Lactobacillus casei and Lactobacillus rhamnosus strains marketed as probiotics. Appl Environ Microbiol 2013, 79:1923-1933.

22. Mandell L, Moran AP, Cocchiarella A, Houghton J, Taylor N, Fox JG, Wang TC, Kurt-Jones EA: Intact gram-negative Helicobacter pylori, Helicobacter felis, and Helicobacter hepaticus bacteria activate innate immunity via toll-like receptor 2 but not toll-like receptor 4. Infect Immun 2004, 72:6446-6454.

23. Moran AP: Lipopolysaccharide in bacterial chronic infection: insights from Helicobacter pylori lipopolysaccharide and lipid A. Int J Med Microbiol 2007, 297:307-319.

24. Kuijf ML, Samsom JN, van Rijs W, Bax M, Huizinga R, Heikema AP, van Doorn PA, van Belkum A, van Kooyk Y, Burgers PC, Luider TM, Endtz HP, Nieuwenhuis EE, Jacobs BC: TLR4-mediated sensing of Campylobacter jejuni by dendritic cells is determined by sialylation. J Immunol 2010, 185:748-755.

25. Stephenson HN, John CM, Naz N, Gundogdu O, Dorrell N, Wren BW, Jarvis GA, Bajaj-Elliott M: Campylobacter jejuni lipooligosaccharide sialylation, phosphorylation, and amide/ester linkage modifications fine-tune human Toll-like receptor 4 activation. J Biol Chem 2013, 288:19661-19672.

26. Sun X, Zhang M, El-Zataari M, Owyang SY, Eaton KA, Liu M, Chang Y, Zou W, Kao JY: TLR2 mediates Helicobacter pylori-induced tolerogenic immune response in mice. PLoS One 2013, 8:e74595.

\section{Submit your next manuscript to BioMed Central and take full advantage of:}

- Convenient online submission

- Thorough peer review

- No space constraints or color figure charges

- Immediate publication on acceptance

- Inclusion in PubMed, CAS, Scopus and Google Scholar

- Research which is freely available for redistribution 